\title{
PAPER
}

\section{Deep brain stimulation of the subthalamic nucleus: effectiveness in advanced Parkinson's disease patients previously reliant on apomorphine}

\author{
T R K Varma, S H Fox, P R Eldridge, P Littlechild, P Byrne, A Forster, A Marshall, \\ H Cameron, K Mclver, N Fletcher, M Steiger
}

J Neurol Neurosurg Psychiatry 2003;74:170-174

See end of article for authors' affiliations

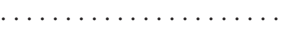

Correspondence to: Dr S H Fox, Walton Centre for Neurology and Neurosurgery, Lower Lane, Fazakerley, Liverpool L9 7LJ UK;

shfox@hotmail.com

Received 15 May 2002 Accepted in revised form 23 October 2002

\begin{abstract}
Objectives: To assess the efficacy of bilateral subthalamic nucleus (STN) deep brain stimulation (DBS) in patients with advanced Parkinson's disease previously reliant on apomorphine as their main antiparkinsonian medication.

Methods: Seven patients with motor fluctuations despite optimal medical treatment given as predominantly apomorphine infusion $(n=6)$, or intermittent apomorphine injections $(n=1)$ underwent bilateral STN DBS using frameless stereotactic surgery. Standard assessments of parkinsonism and motor fluctuations, using Unified Parkinson's Disease Rating Scale (UPDRS) were performed before and six months after surgery. Assessments were performed both on and off medication, and postoperative with the stimulators switched on and off.

Results: Bilateral STN DBS improved motor scores (UPDRS III) by $61 \%$ when off medication $(p<0.05)$. Clinical fluctuations (UPDRS IV items 36-39) were reduced by $46.2 \%(p<0.05)$. Total daily apomorphine dose was reduced by $68.9 \%(p<0.05)$ and apomorphine infusion via a pump was no longer required in four patients. There were no operative complications. Two patients required treatment for hallucinations postoperatively but there was no significant change in mini-mental state examination.

Conclusions: In patients with advanced Parkinson's disease, previously reliant on apomorphine, bilateral STN DBS is an effective treatment to reduce motor fluctuations and enable a reduction in apomorphine use.
\end{abstract}

ong term treatment of Parkinson's disease with levodopa may result in the emergence of motor fluctuations and dyskinesia. ${ }^{1}$ There are a number of strategies used to minimise these complications including the use of directly acting dopamine receptor agonists. In patients with advanced disease in whom oral medication is ineffective or intolerable, intermittent subcutaneous injection of the dopamine receptor agonist, apomorphine can be very effective. ${ }^{2}$ Longer term infusion of apomorphine over several hours a day is often required in patients with marked motor fluctuations. ${ }^{2-4}$ The use of apomorphine by infusion, and thus the management of such advanced patients, varies between movement disorder centres. Because of availability in the UK, there is a higher use of apomorphine infusion compared with centres in North America. Within the UK, our centre, has one of the highest uses of apomorphine.

The main alternative for such advanced patients in centres where apomorphine infusion is not widely adopted is surgical treatment. Currently bilateral deep brain stimulation (DBS) of the subthalamic nucleus (STN) is a recognised treatment option for such patients with advanced Parkinson's disease. In general, studies published from movement disorders centres in America and Europe have shown the benefits of STN DBS in younger patients in whom levodopa and oral dopamine receptor agonists are the main stay of treatment. ${ }^{5-9}$ The series reported by Limousin et al included 10 patients who were using apomorphine before surgery, however, it is not clear if these patients were using intermittent subcutaneous injections or infusion.

To date, there are no case series published reporting the efficacy of bilateral STN DBS in patients with advanced Parkinson's disease all previously requiring apomorphine infusion as the main antiparkinsonian medication. In view of our extensive experience of apomorphine treatment of advanced Parkinson's disease in our centre, we evaluated our experience of bilateral STN DBS in such patients. We present the results of six months follow up on seven patients in whom subcutaneous apomorphine was the principal preoperative antiparkinsonian drug.

\section{METHODS \\ Patients}

Seven patients (all male), mean (SD) age 61.0 (8.1) years, with advanced Parkinson's disease (according to UK Brain Bank criteria $\left.^{10}\right)$, Hoehn and Yahr rating off medication 4-5. and on medication 2.5-4, mean (SD) disease duration 11.6 (4.6) years, were selected for bilateral DBS STN surgery. Patients with a history of cognitive or psychiatric problems were excluded. All patients were levodopa responsive but despite optimal medication were experiencing motor fluctuations and dyskinesia. On average the proportion of the day when the patients were switched "off" was $26 \%-50 \%$ and when switched "on", dyskinesia was present for an average $26 \%-50 \%$ of the day. Median total UPDRS off medication was 120 (range 109-157) and on treatment was 66 (range 53-94).

All patients were using apomorphine as the principal antiparkinsonian medication. Six of the seven patients were receiving apomorphine via subcutaneous infusion (mean (SD) total daily dose 75.7 (45.9) $\mathrm{mg}$ ) over mean 12 hours (range 10-12 h)/day (table 1). Four of these patients also used additional subcutaneous intermittent injections. One of the

Abbreviations: STN, subthalamic nucleus; DBS, deep brain stimulation; UPDRS, Unified Parkinson's Disease Rating Scale; ADL, activities of daily living; MMSE, mini-mental state examination 
Table 1 Individual ant-parkinsonian medication before and six months after bilateral STN DBS implantation

\begin{tabular}{|c|c|c|c|c|c|c|c|c|}
\hline \multirow[b]{2}{*}{ Patient } & \multicolumn{3}{|l|}{ Preoperative } & \multicolumn{5}{|c|}{ Postoperative } \\
\hline & $\begin{array}{l}\text { Levodopa } \\
\text { equivalent } \\
\text { (mg/d) }\end{array}$ & $\begin{array}{l}\text { Apo infusion } \\
\text { (mg/d) }\end{array}$ & Apo sc $(\mathrm{mg} / \mathrm{d})$ & $\begin{array}{l}\text { Levodopa } \\
\text { equivalent } \\
\text { (mg/d) }\end{array}$ & $\begin{array}{l}\text { Apo infusion } \\
\text { (mg/d) }\end{array}$ & Apo sc $(\mathrm{mg} / \mathrm{d})$ & $\begin{array}{l}\text { Percentage } \\
\text { change in daily } \\
\text { levodopa } \\
\text { equivalent }\end{array}$ & $\begin{array}{l}\text { Percentage } \\
\text { change in total } \\
\text { daily } \\
\text { apomorphine }\end{array}$ \\
\hline 1 & 3600 & 150 & 60 & 800 & 100 & 42 & -60.5 & -32.4 \\
\hline 2 & 2350 & 75 & 15 & 980 & 0 & 15 & -58.3 & -83.3 \\
\hline 3 & 1505 & 30 & 3 & 1105 & 0 & 9 & -26.6 & -72.3 \\
\hline 4 & 2775 & 120 & 0 & 1200 & 0 & 0 & -56.8 & -100 \\
\hline 5 & 540 & 45 & 9 & 780 & 45 & 12 & +44.4 & +5.6 \\
\hline 6 & 1100 & 30 & 0 & 400 & 0 & 0 & -63.6 & -100 \\
\hline 7 & 2600 & 0 & 80 & 1500 & 0 & 0 & -42.3 & -100 \\
\hline
\end{tabular}

Apo, apomorphine; sc, subcutaneous. Percentage change is between preoperative and postoperative dose. Levodopa equivalent dose is defined in the text.

seven patients was using apomorphine as intermittent subcutaneous only (total daily dose $60-80 \mathrm{mg}$ ). No patients were experiencing side effects with apomorphine use. Six patients were also using levodopa and oral dopamine receptor agonists (table 1). The mean (SD) total daily levodopa "equivalent" dose of 2067 (1064) mg/d was adapted from Lozano et $a_{l}{ }^{11}$ ( $100 \mathrm{mg}$ levodopa $=10 \mathrm{mg}$ apomorphine $=1 \mathrm{mg}$ pergolide $=3 \mathrm{mg}$ ropinirole). One patient had a left pallidotomy, three years previously. All patients gave written informed consent.

\section{Surgery}

Surgery was performed using a neurosurgical robot (ISS Neuromate). The robot has been shown to have accuracy in clinical use comparable to that of stereotactic frames, mean error $<3 \mathrm{~mm} .{ }^{12}$ The robot is used in a frameless mode, requiring the implantation of skull based fiducials before imaging. Magnetic resonance imaging (MRI) was performed under general anaesthetic on the day before surgery. Axial T2 slices were acquired, with multiple acquisitions to improve image quality. Total scan time for this protocol was about 50 minutes. Images were then transferred to a planning station and reformatted. The STN was directly identified from the images, a trajectory planned to the target from a frontal burr hole, avoiding the ventricles and eloquent structures.

Surgery was performed under local anaesthetic with the patient off medication. To identify the functional target, microelectrode recordings were performed using FHC tungsten microelectrodes. Macroelectrode stimulation (pulse width $60 \mu$ s, frequency $135 \mathrm{~Hz}$ ) was performed once the STN had been identified. Accuracy of the target was assessed by improvement in arm and leg rigidity, bradykinesia (finger taps) and tremor, without inducing side effects. A deep brain electrode was then implanted and externalised for temporary external stimulation. All patients were implanted bilaterally during the same operating period. Five days later a dual channel pulse generator (Kinetra, Medtronic) was implanted into the infraclavicular fossa under general anaesthetic.

Postoperative adjustment to electrical parameters of pulse width, frequency and voltage, electrode contact, and polarity (mono or bipolar) were performed using a console programmer until optimal antiparkinsonian effects were seen, without side effects (table 2). A concomitant change in medication was made according to the effects of stimulation. Adjustments were made as often as required by the patient until a stable effect was achieved both off and on medication.

\section{Clinical evaluations}

All patients were assessed preoperatively, within one month of surgery. Parkinsonian motor scores were assessed using the Unified Parkinson's Disease Rating Scale (UPDRS part III). ${ }^{13}$ The same observer preformed all assessments. Unblinded evaluations were made "off medication" after an overnight withdrawal of all antiparkinsonian medication and "on medication" when the best improvement in motor function after the patient's normal morning dose of antiparkinsonian medication was seen. Activities of daily living (ADL) were assessed using UPDRS part II. Clinical fluctuations and dyskinesia were assessed using UPDRS part IV. Dyskinesia severity was assessed, blind to treatment, by post hoc video analysis using a dyskinesia scale based on rating different body parts. ${ }^{14} \mathrm{~A}$ mini-mental state examination (MMSE) was also performed.

Assessments were repeated at six months after surgery, with stimulation switched on at optimal parameters, in both off and on medication, and after switching off the stimulator for at least 30 minutes, off and on medication. For on medication assessments, the patients received the same dose of morning medication as had been used preoperatively. Patient 1 was assessed at eight months after surgery.

\section{Statistical analysis}

The preoperative and postoperative non-parametric scores for UPDRS motor part III, dyskinesia severity and UPDRS part II ADL were compared using analysis of variance Friedman test and post hoc Dunn's multiple comparison test for variables of surgery (before and after surgery), stimulation (on and off stimulator), and medication (on and off medication) or by the paired Wilcoxon signed rank test. Preoperative and postoperative UPDRS part III subscores for tremor, rigidity, bradykinesia, gait and postural stability, on and off medication, were compared using Wilcoxon signed rank test. UPDRS part IV and MMSE preoperative and postoperative scores were compared using Wilcoxon signed rank test. Antiparkinsonian medication preoperatively and postoperatively was compared using a paired $t$ test. Significance in all cases was assigned when $\mathrm{p}<0.05$.

\section{RESULTS}

\section{Motor scores}

There was a significant improvement in UPDRS part III motor scores at six months postoperatively compared with preoperatively $(p<0.0001$ ) (fig 1 ). Thus preoperative UPRDS part III off medication improved by $61 \%$ postoperatively when on stimulation and off medication $(\mathrm{p}<0.05)$ and by $71 \%$ on stimulation, on medication $(p<0.001)$ (fig 1). There was no significant improvement postoperatively either on or off medication when the stimulator was switched off, compared with preoperative on or off medication ( $p>0.05$, for all conditions). Off medication subscores of the UPDRS part III for tremor, rigidity, bradykinesia, gait, and postural stability were all significantly improved postoperatively, with stimulation on when compared with preoperatively off medication $(\mathrm{p}<0.05$ for all scores, table 3 ). When compared in the on medication 
Table 2 Individual patient stimulator settings at six months after bilateral STN DBS implantation

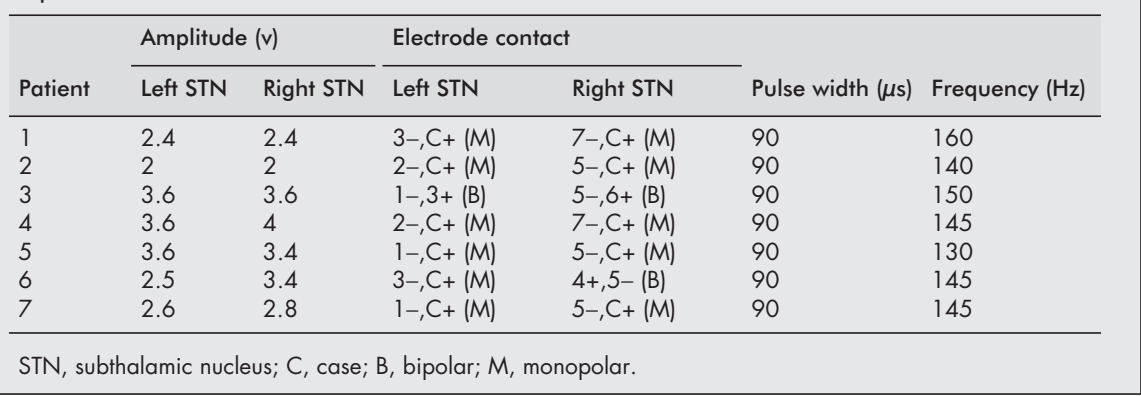

Table 3 Effect of bilateral STN DBS on UPDRS part II activities of daily living (ADL) and part III subscores, both on and off medication. Postoperative scores are all with stimulation on. Data show median (and range), maximum possible scores are also shown. Percentage change is between preoperative and postoperative condition

\begin{tabular}{|c|c|c|c|c|c|c|c|c|}
\hline \multirow[b]{2}{*}{ UPDRS subscale } & \multicolumn{4}{|c|}{ On medication } & \multicolumn{4}{|c|}{ Off medication } \\
\hline & Preoperative & $\begin{array}{l}\text { Postoperative } \\
\text { stimulator on }\end{array}$ & Significance & $\begin{array}{l}\text { Percentage } \\
\text { change }\end{array}$ & Preoperative & $\begin{array}{l}\text { Postoperative } \\
\text { stimulator on }\end{array}$ & Significance & $\begin{array}{l}\text { Percentage } \\
\text { change }\end{array}$ \\
\hline ADL (0-52) & $15(3-33)$ & $14(4-25)$ & $p<0.05$ & $18.7 \%$ & $38(14-43)$ & $25(8-33)$ & $p<0.05$ & $30.6 \%$ \\
\hline Tremor (0-28) & $2(0-20)$ & $0(0-8)$ & NS & $37.1 \%$ & $12(2-26)$ & $2(0-11)$ & $p<0.05$ & $67 \%$ \\
\hline Rigidity (0-20) & $5(0-15)$ & $1(0-10)$ & $p<0.05$ & $72.1 \%$ & 12 (9-15) & $4(0-11)$ & $p<0.05$ & $65 \%$ \\
\hline Bradykinesia (0-32) & $17(6-29)$ & $13(6-22)$ & NS & $10.9 \%$ & $28(21-32)$ & $16(12-31)$ & $p<0.05$ & $36 \%$ \\
\hline Gait $(0-4)$ & $2(0-3)$ & $1(0-3)$ & NS & $23.8 \%$ & $3(1-4)$ & $1(1-3)$ & $p<0.05$ & $50 \%$ \\
\hline Postural stability (0-4) & $2(0-3)$ & $1(0-2)$ & NS & $33.3 \%$ & $4(2-4)$ & $1(1-2)$ & $p<0.05$ & $61 \%$ \\
\hline
\end{tabular}

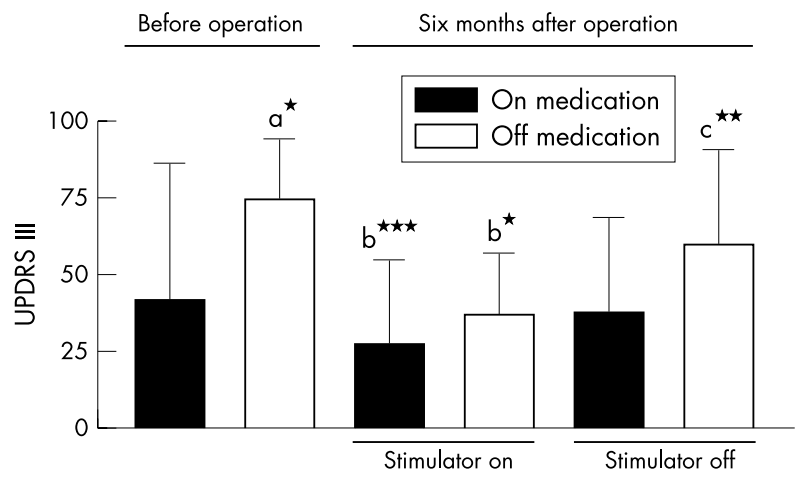

Figure 1 Graph showing median (and range) Unified Parkinson's Disease Rating Scale UPDRS part III motor scores preoperative and six months postoperative after bilateral STN DBS, both on and off medication and with stimulation on and off. Maximum possible UPDRS III score is 108. ${ }^{*} \mathrm{p}<0.05,{ }^{* * *} p<0.001, \mathrm{n}=7$.

$a=$ compared with preoperative on medication; $b=$ compared with preoperative off medication; $c=$ compared with postoperative on medication, stimulator on.

state, preoperatively and postoperatively, only rigidity subscores was significantly improved (table 3 ). There was no significant change in UPDRS part III subscores with the stimulator switched off, both on and off medication compared with preoperatively ( $p>0.05$ for all conditions, data not shown).

\section{Activity of daily living}

UPDRS part II ADL scores, on and off medication, were significantly improved by surgery $(\mathrm{p}<0.001)$. ADL scores were improved by $60.9 \%$ postoperatively, stimulation on, on medication compared with preoperatively off medication $(\mathrm{p}<0.01)$. When compared with respect to medication, postoperative ADL score off medication significantly improved by $30.6 \%$ compared with preoperative off medication $(\mathrm{p}<0.05)$ and postoperative on medication improved by $18.7 \%$ compared with preoperative on medication $(\mathrm{p}<0.05)$ (table 3$)$.

\section{Fluctuations and dyskinesio}

There was a significant reduction in motor fluctuations, including the proportion of the day spent "off", "wearing off", and "sudden, unpredictable offs" after surgery. Thus preoperative clinical fluctuations were reduced by $46.2 \%$ postoperatively (UPDRS part IV, items 36-39, median 5 (range 2-6) and median 2 (range $1-3$ ), p $<0.05$ ). The proportion of the day spent "off" was reduced from median $26 \%-50 \%$ of the day preoperatively to median $0 \%$ postoperatively (UPDRS part IV item 39 median 2 (range $1-3$ ) and median 0 (range 0-1) respectively, $\mathrm{p}<0.05$ ).

Dyskinesia duration and severity was also significantly reduced by $44 \%$ compared with preoperatively (UPDRS part IV, items 32, 33, 34 median 2 (range 1-5) and 6 (range 3-10) respectively, $\mathrm{p}<0.05)$. The proportion of the day with dyskinesia was significantly reduced by bilateral STN stimulation. Thus preoperatively, dyskinesia was present for median $26 \%-50 \%$ of the day compared with median $1 \%-25 \%$ of the day postoperatively (UPDRS part IV, item 32 median 2 (range $1-3$ ) and median 1 (range $1-2$ ) respectively, $p<0.05$ ).

Bilateral STN DBS significantly improved dyskinesia severity scores as assessed by post hoc video analysis $(\mathrm{p}<0.0001)$. Postoperatively with the stimulator on, off medication, dyskinesia was reduced by $85.9 \%$ compared with preoperatively on medication (median dyskinesia 0 (range 0-3) compared with 5 (range 3-20) respectively, $\mathrm{p}<0.05$ ). There was a nonsignificant reduction in dyskinesia by $48 \%$ when compared preoperatively and postoperatively, on medication. When the stimulator was switched off postoperatively and on medication, dyskinesia was significantly reduced by $75.9 \%$ compared with preoperatively on medication (median dyskinesia 0 (range 0-11) compared with 5 (range 3-20), respectively, $\mathrm{p}<0.05)$. 


\section{Medication}

Daily total levodopa equivalent medication was significantly reduced by $37.1 \%$ from 2067.1 (1064.4) $\mathrm{mg} / \mathrm{d}$ to 1055 (379.7) $\mathrm{mg} / \mathrm{d}(\mathrm{p}<0.05)$ (table 1$)$. The apomorphine total daily dose was significantly reduced by $68.9 \%$ from $88.1(62.6) \mathrm{mg} / \mathrm{d}$ to 31.9 (52.6) mg/d $(\mathrm{p}<0.05)$. Four patients no longer required an apomorphine pump postoperatively, two of these patients remained on small doses of apomorphine by intermittent subcutaneous injection (patients 2 and 3). Two patients still required apomorphine pumps postoperatively. In patient 1 the dose was reduced from $150 \mathrm{mg}$ to $100 \mathrm{mg}$, while patient $5 \mathrm{had}$ been on apomorphine alone preoperatively and remained on $45 \mathrm{mg}$ postoperatively with additional oral levodopa (table 1).

\section{Side effects}

Bilateral STN DBS had no significant effect on MMSE $(\mathrm{p}>0.05)$. Thus median preoperative MMSE was 29 (range 29-30) compared with 29 (25-30) postoperative. One patient required early revision of connection wires because of wire fracture within two weeks of surgery. Most patients experienced transient parasthesias after switching on the stimulator. One patient developed disinhibited behaviour, which responded to a reduction in levodopa. Two patients required treatment with quetiapine for visual hallucinations, which developed several weeks after surgery.

\section{DISCUSSION}

This case series confirms the findings of previous reports that bilateral STN DBS is an effective treatment for patients with advanced Parkinson's disease. ${ }^{5-9}$ Significant improvements were seen in all parkinsonian symptoms. In patients previously reliant on apomorphine infusion, this surgical intervention enabled four of six patients either to dispense with apomorphine or to switch from an infusion to a lower dose given as intermittent subcutaneous injections. This has resulted in a great improvement in the patient's and carer's quality of life. Longer follow up of these patients is required to determine if this initial improvement at six months is maintained.

The practice at our centre of using apomorphine infusion as a treatment for motor fluctuations is such that the patients presented here were at a more advanced stage compared with other previously reported groups. Thus the mean (SD) UPDRS part III (motor score) preoperatively off medication was in general higher in our series at 74.3 (10.1) compared with other case series (where figures are available) for example, 59.0 $(10.1),{ }^{5} 55.7(12.1),{ }^{6} 67.6(9.9),{ }^{7} 49.6(14),{ }^{8} 54(15.1) .{ }^{15}$ Despite this, the overall reduction in motor scores UPDRS part III (preoperative off medication compared with postoperative off medication, stimulation on) at $61 \%$ is comparable with other groups where UPDRS part III improved by between $42 \%-71 \%$ after six months. ${ }^{5-9} 15$ Despite the severity of Parkinson's disease in these patients, there was little difference in age at surgery or disease duration compared with other groups. This suggests that bilateral STN DBS is equally effective in these very advanced patients.

In keeping with other studies, there was an overall reduction in medication postoperatively. Of importance in this series, is that four of six patients no longer required apomorphine infusion via a pump and the patient previously reliant on intermittent subcutaneous injections no longer required apomorphine. The two patients still requiring apomorphine via an infusion pump were at a more advanced stage (UPDRS part III off medication scores 94 and 78) and had continued disability despite optimal stimulation. However, improvements in other aspects of daily life were seen that both patients and carers found of benefit; in particular, improved sleep patterns, reduction in drooling saliva, and some improvement in off period pain. ${ }^{5}$ Limousin et al, similarly reported a marked reduction in apomorphine use after surgery, such that only 1 of 10 patients were still requiring apomorphine at low dose $(6 \mathrm{mg} / \mathrm{d})$.

Bilateral STN DBS also resulted in a significant reduction in off period motor fluctuations by $46.2 \%$. The commonest indication for apomorphine use in this series of patients was for off period complications but with variable benefit. Off period pain also responded well to surgery. Two patients required morphine preoperatively that was stopped postoperatively. Thus, in patients where off periods are an important source of disability, bilateral STN DBS seems to be more effective that apomorphine infusion.

In keeping with previous reports, ${ }^{5-9}{ }^{15}$ dyskinesia was also reduced after surgery; both assessed from historical data (UPDRS part IV) and by blinded post hoc video analysis. Of interest, there was a reduction in dyskinesia compared with preoperatively when the stimulator was switched off and after administration of the same preoperative morning antiparkinsonian medication. This would suggest that long term stimulation may "deprime" the patient such that the same dose of levodopa has an equivalent antiparkinsonian action (that is, there was no significant change in UPDRS part III between preoperative on medication and postoperative on medication, stimulation switched off) but there is less dyskinesia after bilateral STN DBS. This finding has been previously noted and discussed by other centres. ${ }^{16}{ }^{17}$ Dyskinesia reduction after bilateral STN DBS is thought to be primarily attributable to a concomitant reduction in medication. However, there is probably an effect on basal ganglia circuitry in addition whereby continuous STN DBS changes neural pathways responsible for the generation of levodopa induced dyskinesia.

No formal cognitive testing was performed in this series but median MMSE score was unchanged. Two patients developed hallucinations postoperatively that did not respond to reduction of antiparkinsonian medication and required additional treatment with the atypical anti psychotic agent, quetiapine. Despite the advanced stage of Parkinson's disease in our cohort of patients, no other cognitive problems were encountered. However, in view of accumulating evidence that cognitive problems may occur particularly in patients over 70 years, ${ }^{18}$ practice in our centre has now changed to include a full neuropsychological evaluation before surgery.

The neural mechanisms underlying Parkinson's disease involve overactivity of the STN. ${ }^{19-21}$ Levodopa and dopamine receptor agonists, including apomorphine, reduce overactivity of the STN and thus alleviate parkinsonian symptoms. ${ }^{22}$ Bilateral STN DBS is thought to also reduce STN overactivity, although the exact mechanism of action is as yet unknown. ${ }^{24} 25$ Thus both medical and surgical treatment, by reducing overactivity of the STN, will alleviate parkinsonism. Continuous daily apomorphine infusion would be thought to have similar antiparkinsonian effects to continuous bilateral STN DBS. A study measuring cortical inhibition following a single dose of apomorphine and STN stimulation has shown comparable effects. ${ }^{26}$ Our study would suggest that bilateral STN DBS may be superior to apomorphine infusion, but this may simply relate to the continuous 24 hour DBS as compared with between 10 tol2 hours apomorphine. However, to date, the antiparkinsonian effects of apomorphine infusion and bilateral STN DBS surgery have not been compared in a randomised clinical trial. A multicentre study is currently underway in the UK (PD-SURG) in which Parkinson's disease patients in whom there is uncertainty as to whether surgical treatment is the next option are randomised to surgery (bilateral STN DBS) or best medical treatment and deferred surgery for 12 months. Patients randomised to the medical treatment arm may receive apomorphine infusion. This study may therefore permit such a comparison of bilateral STN DBS and apomorphine infusion including the cost effectiveness of such treatment options. 


\section{ACKNOWLEDGEMENTS}

The help of Dr Rhys Davies, Dr Richard Nicholas and the staff on the Walton Centre clinical trials unit is gratefully appreciated.

\section{Authors' affiliations}

T R K Varma, S H Fox, P R Eldridge, P Littlechild, * P Byrne,

A Forster, A Marshall, ${ }^{\dagger} \mathrm{H}$ Cameron, $\mathrm{K}$ Mclver, $\mathrm{N}$ Fletcher,

M Steiger, Walton Centre for Neurology and Neurosurgery, Liverpool, UK

*Current address: Department of Neurosurgery, INS, Southern General Hospital, 1345 Govan Road, Glasgow

${ }^{\dagger}$ Current address: Department of Neurophysiology, Hope Hospital, Stott Lane, Manchester

Competing interests: none declared.

\section{REFERENCES}

1 Nutt JG. Levodopa-induced dyskinesia: review, observations, and speculations. Neurology 1990;40:340-345.

2 Stibe CM, Lees A, Kempster PA, et al. Subcutaneous apomorphine in parkinsonian on-off oscillations. Lancet 1988;i:403-6.

3 Obeso JA, Grandas F, Vaamonde J, et al. Apomorphine infusion for motor fluctuations in Parkinson's disease. Lancet 1987;346: 1376-7.

4 Frankel JP, Lees AJ, Kempster PA, et al. Subcutaneous apomorphine in the treatment of Parkinson's disease. J Neurol Neurosurg Psychiatry 1990;53:96-101.

5 Limousin $P$, Krack $P$, Pollak $P$, et al. Electrical stimulation of the subthalamic nucleus in advanced Parkinson's disease. N Engl J Med 1998;339:1 105-1 1

6 Kumar R, Lozano AM, Kim YJ, et al. Double-blind evaluation of subthalamic nucleus deep brain stimulation in advanced Parkinson's disease. Neurology 1998;51:850-5.

7 Moro E, Scerrati M, Romito LM, et al. Chronic subthalamic nucleus stimulation reduces medication requirements in Parkinson's disease. Neurology 1999:53:85-90.

8 Rodriguez-Oroz MC, Gorospe A, Guridi J, et al. Bilateral deep brain stimulation of the subthalamic nucleus in Parkinson's disease. Neurology 2000;55 (suppl 6):45-51

9 Molinuevo JL, Valldeoriola F, Tolosa E, et al. Levodopa withdrawal after bilateral subthalamic nucleus stimulation in advanced Parkinson disease. Arch Neurol 2000:57:983-8.

10 Hughes AJ, Daniel SE, Kilford L, et al. Accuracy of clinical diagnosis of idiopathic Parkinson's disease: a clinico-pathological study of 100 cases. J Neurol Neurosurg Psychiatry 1992;55:181-4.

11 Lozano AM, Lang AE, Galvez-Jimenez N, et al. Effect of GPi pallidotomy on motor function in Parkinson's disease. Lancet 1995;?:1383-7.
12 Varma TRK, Eldridge PR, Forster AR, et al. Frameless stereotactic surgery for movement disorder using the Neuromate Robot. J Stereotact Funct Neurosurg 2001;77:142.

13 Fahn S, Elton RL, members of the UPDRS Development Committee. The Unified Parkinson's Disease Rating Scale. In: Fahn S, Marsden CD, Calne $\mathrm{DB}$, et al, eds. Recent developments in Parkinson's disease. Flotham Park, NJ: Macmillan Healthcare Information, 1987:153-63.

14 Hagell P, Widner H. Clinical rating of dyskinesias in Parkinson's disease: use and reliability of a new rating scale. Mov Disord 1999;3:448-55.

15 The Deep Brain Stimulation for Parkinson's Disease Study Group Deep-brain stimulation of the subthalamic nucleus or the pars interna of the globus pallidus in Parkinson's disease. N Engl J Med 2001;345:956-63.

16 Bejiani BP, Arnulf I, Demeret S, et al. Levodopa-induced dyskinesias in Parkinson's disease: is sensitisation reversible? Ann Neurol 2000;47:655-8

17 Fraix V, Pollack P, Van Blercom N, et al. Effect of subthalamic nucleus stimulation on levodopa-induced dyskinesia in Parkinson's disease. Neurology 2000;55:1921-3.

18 Saint-Cyr JA, Trépanier LL, Kumar R, et al. Neuropsychological consequences of chronic bilateral stimulation of the subthalamic nucleus in Parkinson's disease. Brain 2000;1 23:2091-108.

19 Mitchell IJ, Clarke CE, Boyce S, et al. Neural mechanisms underlying parkinsonian symptoms based upon regional uptake of 2-deoxyglucose in monkeys exposed to 1-methyl-4-phenyl-1,2,3,6-tetrahydropyridine. Neuroscience 1989:32:213-26.

20 Bergman H, Wichmann T, DeLong MR. Reversal of experimental parkinsonism by lesions of the subthalamic nucleus. Science 1990;249: 1436-8.

21 Obeso JA, Rodriguez-Oroz MC, Rodriguez M, et al. Pathophysiologic basis of surgery for Parkinson's disease. Neurology 2000;55:S7-12.

22 Kreiss DS, Mastropietro CW, Rawji SS, et al. The response of subthalamic nucleus neurons to dopamine receptor stimulation in a rodent model of Parkinson's disease. J Neurosci 1997;17:6807-19.

23 Hassani OK, Feger J. Effects of intrasubthalamic injection of dopamine receptor agonists on subthalamic neurons in normal and 6-hydroxydopamine-lesioned rats: an electrophysiological and c-Fos study. Neuroscience 1999; 92:533-43.

24 Ashby P. What does stimulation in the brain actually do? Prog Neurol Surg 2000;15:236-45

25 Benazzouz A, Hallett M. Mechanisms of action of deep brain stimulation. Neurology 2000;55 (suppl 6):13-16.

26 Pierantozzi M. Palmieri MG, Mazzone $P$, et al. Deep brain stimulation of both subthalamic nucleus and internal globus pallidus restores intracortical inhibition in Parkinson's disease paralleling apomorphine effects: a paired magnetic stimulation study. Clin Neurophysiol 2002;113:108-13 